Cahiers de recherches médiévales

Figures mythiques médiévales auX $\mathrm{XIX}^{\mathrm{e}}$ et $\mathrm{XX} \mathrm{X}^{\mathrm{e}}$ siècles

\title{
La Babel intérieure
}

(Charles d'Orléans et la pluralité des langues)

Alice Planche

\section{CpenEdition}

Journals

Édition électronique

URL : https://journals.openedition.org/crm/1873

DOI : $10.4000 / \mathrm{crm} .1873$

ISSN : 1955-2424

Éditeur

Honoré Champion

Édition imprimée

Date de publication : 15 décembre 2004

Pagination : 231-236

ISSN : $1272-9752$

Référence électronique

Alice Planche, «La Babel intérieure », Cahiers de recherches médiévales [En ligne], 11 | 2004, mis en ligne le 10 octobre 2007, consulté le 15 décembre 2022. URL : http://journals.openedition.org/crm/ 1873 ; DOI : https://doi.org/10.4000/crm.1873

Ce document a été généré automatiquement le 15 décembre 2022.

Tous droits réservés 


\title{
La Babel intérieure
}

\author{
(Charles d'Orléans et la pluralité des langues)
}

\section{Alice Planche}

Comme la langue en général, la multiplicité des parlers est la meilleure et la pire des choses : richesse de l'humanité, arc-en-ciel de la pensée, elle est aussi, à l'évidence, l'obstacle majeur à la communication directe entre les individus et les groupes... Voyages, commerce, diplomatie, liens personnels, guerre même exigent que les parties en présence percent le mur de l'isolement: on ne peut, comme il a été dit, considérer les autres comme des muets ${ }^{1}$. Évidentes en nos temps de mondialisation, ces difficultés ne l'étaient pas moins lorsque les nations n'avaient pas encore de langue officielle, beaucoup se contentant de leurs dialectes.

Dans cette situation, trois voies de salut s'offrent : adopter un medium universel, être soi-même plurilingue, ou recourir, aux services des interprètes - aujourd'hui relayés par les lexiques, dictionnaires et informatique.

Charles d'Orléans pouvait envisager ces trois possibilités. À la différence de certains princes plus formés aux armes qu'aux lettres, il a étudié le latin, celui de Rome et du Vatican, celui des clercs, des juristes, des théologiens, de certains poètes. Il a composé une Ballade ${ }^{2}$ évoquant Noël, dans un latin plié au mètre et aux rimes de chez nous, farci plusieurs pièces de termes ou d'expressions latines, et selon certains critiques, il pourrait être l'auteur des six cents vers du Canticum Amoris, dont les deux manuscrits sont probablement ou, pour l'un, copié par lui, pour l'autre, connu de lui ${ }^{3}$. Sans prendre parti dans cette discussion, il faut reconnaître que ni les cours, ni le peuple n'usent du latin dans leurs échanges: en témoignent l'activité des traducteurs et adaptateurs, surtout à partir du XIV ${ }^{\mathrm{e}}$ siècle. Et, dans les rondeaux écrits après son retour en France, le poète paraît détaché de cette langue, comme s'il l'avait laissée en exil. Ayant traité ailleurs ${ }^{4}$ de cette question, je me contente de rappeler les derniers vers d'une pièce, apostrophant saint Valentin, qui cette année-là ne lui a rien apporté sauf :

... (Fors) bona dies en latin,

Vielle relique en viel satin. (rondeau 276, vv. 10-11)

Le français, qui avait suivi Guillaume le Conquérant à la cour d'Angleterre, avait failli prendre la relève, ayant été un temps «la parlure la plus commune à toute gens" 
jusqu'à ce que la guerre, la peste et les bandes armées le fassent se replier. Ni l'Italie malgré son prestige, ni aucune création artificielle n'avaient ce statut.

5 C'est en partie pourquoi les 'polyglottes' - avant la lettre ${ }^{6}-s^{\prime}$ étaient multipliés. Les échos littéraires en témoignent ; mais bornons-nous ici à Charles d'Orléans qui, par sa naissance, sa culture, son rôle de prince, sa captivité de vingt-cinq ans Outre-Manche, avait toutes raisons d'entendre et de tenter de comprendre divers idiomes. Combien ? Dans quelle mesure? Sur ce problème, je me contenterai d'un écho, dans une de ses chansons, sous un jour assez sombre :

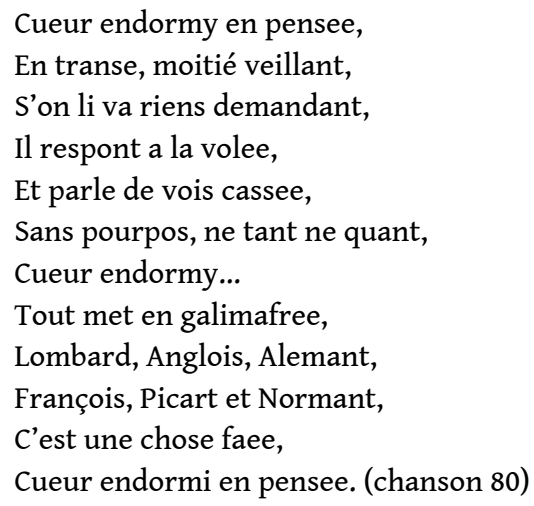

6 Le poète connaît bien - trop bien à son gré - ces états incertains, intermédiaires entre sommeil et veille, alors que le contact avec ce qui nous environne, gens et choses, est presque rompu, sans que la conscience soit tout à fait éteinte. L'ancien et le moyen français disposaient de mots perdus pour traduire l'intraduisible. Ainsi Chrétien de Troyes et le Villon du Lais ${ }^{7}$ utilisaient le terme " entroubli » et le verbe " entroublier " (" entre » ouvrait alors toute une série d'expressions). Le nom " dorveille " n'apparait plus guère après le XIV ${ }^{e}$ siècle. S'il ne fait pas appel à ce lexique, Charles d'Orléans se livre à une sorte d'analyse qui renvoie à une conception plurielle de l'être, vu moins comme un individu que comme un faisceau d'instances distinctes, en accord ou en lutte. Tels sont la "pensee " (participe passé substantivé), le "penser» (infinitif plus actif), le "cueur », vulnérable, instable, au centre des figures, ailleurs les yeux et les oreilles, quand ce n'est pas les yeux et les oreilles du cueur. Reste celui qui dit «je », qui est nommé « moi », comme ici :

Mon cueur, Penser et moi, tous trois... (ballade 98, v. 9)

7 Dès l'incipit de la Chanson 80 , le cœur calmé est enveloppé, comme bercé par la pensée qui fait écran aux sollicitations du monde. Cependant ce cœur n'est pas plongé dans un sommeil total, il est en transe, ce qui n'a rien à voir avec le sens alors courant d'être en train de mourir et d'aller chez les transis - aujourd'hui les trépassés -, c'est, dirionsnous, être en transit, en transition entre présence et absence: s'il entend qu'on l'interpelle, il ne saisit pas le sens de la question, à peine un mot auquel il répond «à la volée » (v. 4) comme un mauvais joueur de paume relance la balle reçue au hasard, sans viser aucun but. Ici à la volée n'évoque en rien l'essor de l'oiseau, seulement un geste maladroit, vu du dehors. Et c'est aussi comme du dehors que le cœur entend sa propre voix « cassée ", sans force, à peine audible, le pouvoir de parler clair s'étant effacé en même temps que la possibilité de comprendre les autres.

C'est alors qu'intervient, dans l'allusion, la présence d'une Babel toute intime ${ }^{8}$, puisque c'est en lui-même que le poète constate ce que les témoins pourraient confirmer: l'indistinction des parlers qu'il manie ou a maniés plus ou moins longtemps, plus ou 
moins bien au cours de sa vie. Tels sont le lombard de sa mère, pourtant fille de France, mais aussi née de Galeas Visconti ; l'anglais qu'il a subi pendant les vingt-cinq ans de sa captivité, dans lequel il a composé au moins deux pièces, pour certains plus ${ }^{9}$, et qu'à son retour il aurait su mieux que sa langue; puis l'allemand, du moins celui des bords du Rhin, à la limite de l'actuelle Hollande, dans le duché de Juliers et de Berg, à Clèves, dont sa troisième épouse, Marie, porte le nom ${ }^{10}$

Trois dialectes d'oïl : le français de Paris et des bords de Loire, le picard voisin et naguère rival littéraire, le normand, dont est issu l'anglo-normand, idiome officiel de la cour et de l'administration anglaises à la prise de pouvoir de Guillaume le Conquérant et dont, malgré la guerre, il restait des traces... Ces derniers parlers, proches mais distincts, et dont les hôtes de Blois devaient garder l'accent, nous rappellent que la France était encore loin du medium unifié et officiel dont l'Édit de Villers-Cotterets imposera l'usage en 1539. Les limites parfois imprécises des langues, acquises ou entendues à des époques diverses par le prince, ont contribué à former ce ragoût indigeste, désigné ici par le terme expressif et comique de "galimafrée », d'origine incertaine, peut-être fait du verbe "galer » et de " mafrer ", " manger gouluement ", en picard, venu du moyen hollandais. Mais, l'étymologie mise à part, on pense à galimatias, galipette, galéjade, appelant le même sourire. Le résultat semble l'effet d'un sort, il est «faé » (de fata, comme fée) ce qui n'a pas d'équivalent aujourd'hui, où « enchanté » a pris une valeur positive.

10 La Chanson 80 n'utilise ni le je, ni aucun adjectif ou pronom de la première personne: l'épreuve est dépersonnalisante. Au contraire, le singulier et obscur rondeau 211, qui évoque aussi l'éventail des parlers fait appel à toute la gamme de termes désignant la première personne du singulier, « je » $y$ compris :

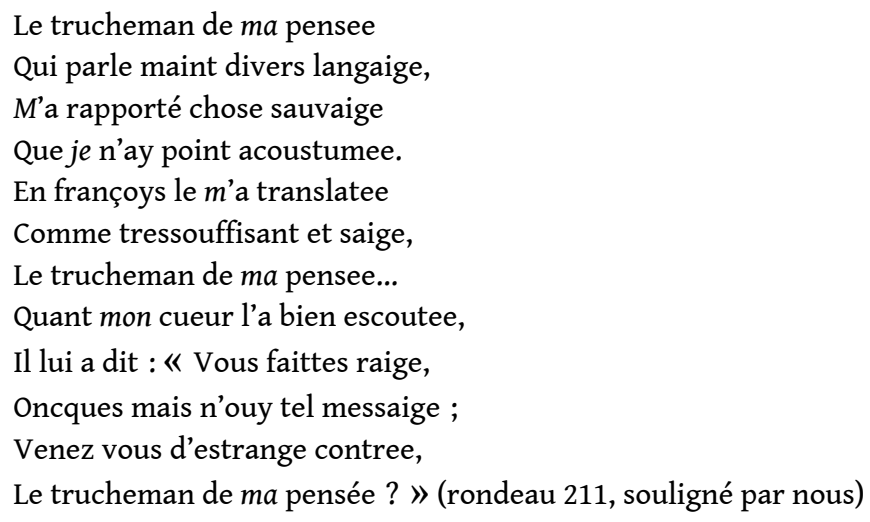

Il s'agit d'une expérience, vécue de façon consciente, dans l'éveil et l'attention de tout l'être, dont le je totalisant.

L'incipit a probablement été lancé à la cour de Blois par le prince lui-même; utilisé deux fois par lui (R. 210-211) et par deux de ses proches, comme il était alors assez courant à Blois, entre autres dans les variations sur le quasi-proverbe inspiré du supplice de Tantale : «Je meurs de soif auprès de la fontaine... $»^{11}$.

13 Mais cette fois le nom initial relève du lexique des cours et des marchés, lieux où se côtoient, pour des échanges, les gens venus de pays aux parlers divers. Malgré ses efforts de plurilinguisme, Charles d'Orléans a eu recours aux services de ces intermédiaires, en Angleterre et en France. "Trucheman ", apparut dès le XI siècle sous des formes variées («drugman», «drugeman »...) venues du turc tourguman. Le retour du poète en France correspond à une partie du règne de Mehmet II qui, avant 
Soliman le Magnifique, étendit son royaume et, en 1453, conquit Constantinople. Bien avant, les souverains turcs envoyaient des missions vers la Chrétienté et avaient besoin d'être compris, donc d'avoir des turguman, d'où l'origine du terme.

Ici la métaphore s'intériorise. La vie intime est vécue comme lourde de ces profondeurs obscures, qu'illuminent des éclairs de lucidité qui, le plus souvent, faute d'un effort pour trouver un vocabulaire tout prêt, s'éteignent et s'oublient. Par un effort d'attention que note la comparaison avec les traducteurs spécialisés, le "sauvage » est changé en "souffisant» et en "sage». Sauvage ne signifie ni rude ni grossier, mais venu d'un monde inexploré comme le fut longtemps la forêt, la silva latine, terme qui a donné silvaticus, en bas latin salvaticus, en français "sauvage». Ainsi Dante, aux premiers vers de la Divine Comédie, se retrouve dans un état second, dans la "selva oscura... salvaggia $»^{12}$, avant que lui apparaissent les horreurs de l'enfer.

Le passage à la langue commune ne suppose aucune perte. "Souffisant» n'a aucune connotation négative, c'est ce qui suffit, ce qui convient ; "sage » est à la fois savant dans la science du déchiffrage, et réfléchi dans l'interprétation.

Après les réactions du Je totalisant, le cœur, partie la plus tendre de la personne, manifeste une surprise encore plus marquée, que note d'abord «faire rage». Ce n'est pas ici d'un délire furieux qu'il est question, mais plutôt d'une prouesse dépaysante, révélant une origine lointaine et un caractère neuf, non ouï (le composé « inouï » ne sera en usage qu'au XVI e siècle). "Étrange " signifie d'abord " étrangère ", avec une légère connotation de singularité, sens qui aujourd'hui l'a emporté.

Pour mesurer ce que ce rondeau a d'original, on peut le comparer avec les trois autres sur le même début. Le rondeau 115 de Frédet est une banale déclaration faite à une dame le jour de la Saint-Valentin. Le rondeau 212 du Comte de Clermont, sans référence de date, a à peu près le même sens. Reste le numéro 210 , de Charles d'Orléans, luimême, beaucoup plus riche et complexe. Le traducteur y est présenté comme l'envoyé de Réconfort son seigneur ; il arrive auprès du cœur muni d'une lettre de créance. Le cœur ne répond pas aussitôt. Il semble bien encore qu'il s'agisse de sentiments dont la nature précise ne sera définie qu'après un temps de réflexion.

Le rondeau 33 ne fait appel à aucun «truchement »; c'est le cœur lui-même qui, dans un effort douloureux, met au clair et fixe, pour le Livre de Pensée :

... la vraye histoire de douleur

De larmes toute enluminée... (vv. 3-4)

La poésie est à la fois instrument et résultat de la recherche ; l'œuvre devient le journal intime, ce qui fut, bien avant le romantisme, l'un des buts du lyrisme. Du Bellay, par exemple, le précise dans le Sonnet 1 de ses Regrets. L'ambition de ses vers n'a rien d'objectif ou de philosophique, il veut se confier à eux :

... Aussi ne veux je tant les pigner et friser,

Et de plus brave nom ne les veux deguiser

Que de papiers journaux ou bien de commentaire. (vv. 12-14)

Ce rôle de confident est direct, il ne nécessite pas de "truchement ». L'originalité du Rondeau 211 réside dans l'existence d'un intermédiaire interne, saisissant et fixant ce qui, sans lui, resterait dans la nuit, ou n'apparaîtrait que dans un instant de lucidité suivi d'oubli. L'inconnu, ou l'objet d'une illumination vite éteinte, devient ainsi révélation. Cette mise au jour des strates enfouies de l'individu est, bien avant la lettre, une «psychanalyse » qui n'a pas besoin d'un psychiatre étranger, ni d'une théorie 
préalable pour découvrir sa clé, laquelle ne prétend pas « ouvrir toutes les serrures ", comme le dit ironiquement Julien Gracq ${ }^{13}$ à propos du freudisme.

21 Ce qui fait l'objet du poème n'est pas la chose découverte - d'autres vers la disent peutêtre - c'est le fait même de la découvrir : ainsi sommes-nous arrêtés au bord du secret. Cette attente non comblée contribue aussi à la singularité du rondeau.

Du banal constat de l'obstacle à la communication que représente la diversité des langues, une chanson et un rondeau tirent des conséquences sans lien, presque opposées; là l'image de la confusion mentale, ici celle du rôle d'un "truchement " intérieur. Ainsi les objets et les actes courants, évidents, eau vive, forêt, fleurs, ermitage, chasse, navigation, réunion, musique, danse, etc. servent de base et de comparant à la fluidité du moi. Le va-et-vient entre les deux domaines donc aucun n'efface tout à fait l'autre est favorisé par le choix de la forme fixe et du mètre. La reprise d'un refrain - le premier vers - n'est pas une simple fermeture du texte, mais une spirale qui enrichit le sens. Le retour de sonorités, bien au-delà de la rime, le choix de l'impair pour la Chanson 80, l'impair « Plus vague et plus soluble dans l'air... $»^{14}$, puis du pair (octosyllabique) pour le rondeau, contribuent au sens et à la transmission des sentiments, à condition que le lecteur - ou plutôt l'auditeur car il faut écouter plutôt que lire - soit attentif et ouvert. Ces deux pièces ne sont ni les plus claires ni les plus connues de l'œuvre. Elles font partie des plus personnelles.

\section{NOTES}

1.Les Russes ont jadis baptisé les Allemands du nom de niémetz, « muets », qui leur et resté.

2.Toutes les références relatives au poète renvoient à : Charles d'Orléans, Poésies, éditées par P. Champion, Paris, Champion, CFMA 34 et 56. Plusieurs rééditions.

3.Voir G. Ouy, Recherches sur la librairie de Charles d'Orléans et de Jean d'Angoulème. C.R. de l'Académie des Inscriptions et Belles-Lettres, Paris, 1955.

4.Planche A., Charles d'Orléans ou la Recherche d'un langage, Thèse, Paris, Champion, 1975, Bibliothèque du $\mathrm{XV}^{\mathrm{e}}$ siècle $\mathrm{n}^{\circ} 38$.

5.Comme le dit entre autres Marco Polo, qui écrit en franco-vénitien son Devisement du Monde (dit aussi Le Million et Le Livre des Merveilles).

6.Le terme polyglotte n'a été relevé qu'au XVI siècle.

7.François Villon, Le Lais, v. 281.

8.Le terme Babel est absent du vocabulaire poétique de Charles d'Orléans.

9.En particulier cinq rondeaux dont l'attribution est discutée.

10.Marie de Clèves avait cependant été élevée à la cour de Bourgogne où on parlait français.

11.Douze ballades dont deux de Charles d'Orléans (variantes dans le refrain).

12.Dante Alighieri, La Divine Comédie, Chant I, vers 2 et 5.

13.J. Gracq, En lisant, en écrivant...

14.Selon le vers de Paul Verlaine : Paul Verlaine, Jadis et Naguère, Art Poétique, vers 3. 